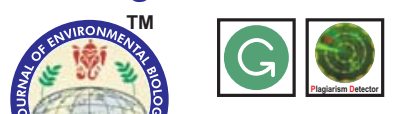

\title{
Assessment of oxidative changes in tobacco seed oil stored at different conditions
}

Authors Info

K. S. Raju ${ }^{1 *}$, D. D. Reddy', H. Ravisankar', T.G.K. Murthy and N.S. Murthy ${ }^{2}$

${ }^{1}$ Central Tobacco Research Institute, Rajahmundry-533 105, India

${ }^{2}$ Godavari Institute of Engineering and Technology,

Rajahmundry-533 105, India

*Corresponding Author Email : rajuks2002@yahoo.co.in

Key words

Conjugated dienes, Oxidative changes, Tobacco seed oil

Publication Info

Paper received: 14.10 .2015 Revised received: 21. 12. 2015 Re-revised received: 30. 07. 2016 Accepted:12. 09. 2016

\section{Abstract}

Aim: Alternative uses of tobacco have gained importance in recent times to sustain crop for nonconventional and economically viable application in food and industries. One of the alternative promising use of tobacco is seed oil having nutritive, pharmaceutical and industrial utility.

Methodology: Experiments were conducted to study the extent of oxidative alterations in tobacco seed oil subjected to ambient and sunlight storage, for a period of 90 days and compared with sunflower and groundnut oils. The magnitude of oxidative changes was monitored by periodical measurement of peroxide value (PV), free fatty acids (FFA), $p$-anisidine value (pAV), conjugated trienes (CT), conjugated dienes (CD) and iodine values (IV).

Results: Peroxide values significantly increased from 15 days of storage $\left(1.13 \mathrm{meq} \mathrm{kg}^{-1}\right)$ reaching maximum value $\left(8.45 \mathrm{meq} \mathrm{kg}^{-1}\right)$ at 90 days. Photo-oxidation $\left(4.82 \mathrm{meq} \mathrm{kg}^{-1}\right)$ was significantly higher than auto-oxidation ( $\left.3.80 \mathrm{meq} \mathrm{kg}^{-1}\right)$. The peroxides were significantly different among the three oils with the highest in tobacco seed oil ( $\left.5.06 \mathrm{meq} \mathrm{kg}^{-1}\right)$ followed by sunflower oil ( $4.29 \mathrm{meq} \mathrm{kg}{ }^{-1}$ ) and groundnut oil (3.58 meq $\mathrm{kg}^{-1}$ ). The pAV increased significantly with increase in days of storage attaining maximum value of 13.84 at 90 days. pAV in tobacco seed oil (7.27) and sunflower oil (7.18) were at a par and were significantly higher than groundnut oil (5.36). Tobacco oil at 90 days of storage showed $2.9 \%$ and $7.19 \%$ higher content of $\mathrm{CD}$ over sunflower oil in photo and auto-oxidations respectively. FFA content in tobacco seed oil $(0.153 \%)$ and sunflower oil $(0.150 \%)$ were at a par and were significantly higher than groundnut oil.

Interpretation: The oxidative changes in tobacco seed oil showed nearly similar trends with that of sunflower oil. The results showed that even though tobacco seed oil contains higher levels of unsaturated fatty acids, tobacco oil can be stored like any other edible oils.

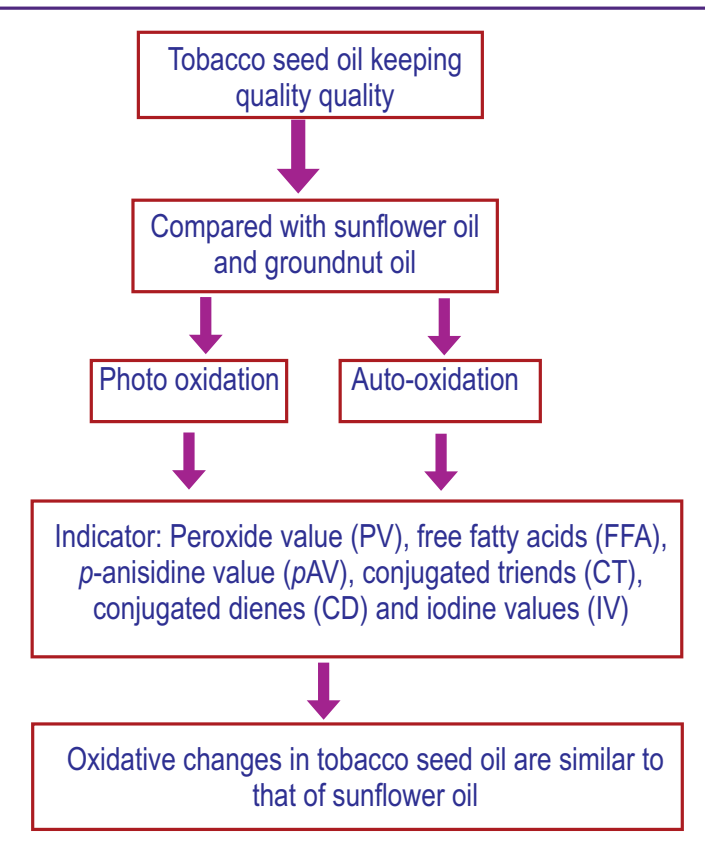




\section{Introduction}

Tobacco is a leading commercial crop in India and is grown in $0.45 \mathrm{M}$ ha area with $750 \mathrm{M} \mathrm{kg}$ leaf production. However, the anti-tobacco campaign for traditional form of consumption is posing a serious challenge to tobacco production, trade and industry. The economic life of millions of people, including six million farmers, depends on tobacco necessitating the crop to be sustained for its potential alternative uses. The promising alternative use of tobacco is seed oil having nutritive, pharmaceutical and industrial uses (Awolola et al., 2010). The research on alternative uses of tobacco is the order of the day, leading to critical examination of potentials of tobacco as 'an oil seed crop'. An estimated 1300-1500 tonnes of tobacco seed oil is expelled and exported from India to other countries for utilization in paint industry (Deo Singh and Narasimha Rao, 2005). It is used as raw material for preparation of printing inks, dyes (Zlatanova et al., 2007), potential use in food and coating industries (Muktha et al., 2007), production of soaps, shoe polish, varnishes (Purseglove, 1991) and an alternative to diesel fuel (Giannelos et al., 2002). However, tobacco seed oil finds extensive use in paints, varnishes, lubricants and soap industries. There has been a significant gap between demand and supply of edible oil because of limited oil seeds and shifting of acreage to other crops, thus making need to search for alternative sources of edible oils.

Tobacco seed contains 35\% oil and linoliec acid is the major fatty acid (66 - 76\%) (Siva Raju et al., 2011). Tobacco seed oil shows promise as edible oil due to demonstration of the dietary effect of linoleic acid rich oils like corn or safflower oils in lowering the serum cholesterol (Zlatanova et al., 2007). Different variables involved in oil shelf-life, such as processing, storage conditions, light exposure, type of packaging material, availability of oxygen, and addition of antioxidants affect the quality of vegetable oils/ fats. Oxidation of oil may be the primary cause of deterioration in quality, and peroxidation is considered a principal mean of deterioration in oil quality. Peroxidation imparts rancid and undesirable flavors to fat products (Pezzuto and Park, 2002). Warm ambient temperature encourage the onset of oxidation in a silent way. Auto-oxidation proceeds through free radical chain reaction by the attacking the double bond at room temperature. Photo-oxidation on the other hand is a much faster reaction that involves attack at double bond by a singlet ${ }^{1} \mathrm{O}_{2}$ species (Eunok Choe and David, 2006). Photo-oxidation postulates that double bond within a fatty acid molecule may be capable of capturing outside source of energy, such as heat and light, to reach a critical excitation level (Fekamrhobo and Obomanu, 2009) and the double bond may break giving rise to a free radical species, which may in turn generate more free radicals. Auto and photooxidation, which are natural oxidation and chemical degradation processes of edible oils, also results in rancidity of food items, resulting these processes to convert fatty acid esters of oils into free fatty acids. The present study was undertaken with an objective to evaluate the extent of oxidative deterioration and shelf-life of tobacco seed oil stored under ambient and sunlight conditions and compared with edible sunflower and groundnut oils.

\section{Materials and Methods}

Tobacco seed, sunflower and groundnut oils were extracted by Soxhelt method using hexane and decolorized by Fullers earth. Five grams of seed was pounded well using mortar and pestle and packed into Whatman thimble. A piece cotton was placed at the top to evenly distribute the solvent as it droped on sample during extraction. Thimbles were placed in butt tubes of the Soxhlet extraction apparatus. The extraction was done with $150 \mathrm{ml}$ hexane for $12 \mathrm{hrs}$ by gentle heating. After extraction, flasks were cooled and hexane was removed using flash evaporator (vacuum evaporator). The flasks were dried in the oven to remove traces of hexane at $70^{\circ} \mathrm{C}$ and the flasks were cooled in the desiccators and weighed. The process was repeated till constant weight of oil was obtained. The samples were stored in $250 \mathrm{ml}$ translucent PET bottles under controlled setup. Two sets of each samples were separately subjected to auto-oxidation (at ambient temperature in dark) and photo-oxidation (exposure to sun light daily for 7 hours) for a period of 90 days. Analysis was carried out at 15 days interval. Oil samples were analyzed for peroxide value, iodine value, (Cox and Pearson, 1962) and free fatty acids (Lowry and Tinsley, 1976). Conjugated dienes and trienes, in terms of specific extinctions at 232 and $268 \mathrm{~nm}$ were determined by spectrophotometer. Oil samples were diluted with isooctane to bring the absorbance within limits $(0.2-0.8)$ and $\varepsilon^{1 \%} 1 \mathrm{~cm}$ was calculated following IUPAC method (IUPAC, 1987). Determination of $p$-anisidine value of the oil samples was determined following IUPAC method (IUPAC, 1987). Samples dissolved in isooctane were allowed to react with $p$-anisidine solution in acetic acid $(0.25 \% \mathrm{w} / \mathrm{v})$ for $10 \mathrm{~min}$ to produce a colored complex, and the absorbance value was noted at $350 \mathrm{~nm}$ by spectrophotometer. Statistical analysis was done by SAS 9.3 software.

\section{Results and Discussion}

Peroxide value (PV) is a sensitive indicator of early stages of oxidative deterioration of fats and oils. The analysis of variance for storage period, oxidation methods and oils was significant for peroxide value (PV). The co-efficient of variation for PV was 8.43 which was reasonably low and correlation coefficient was 0.99 (Table 1). The storage periods, 0 and 15 days were at a par whereas others were significant. Peroxide values significantly increased from 15 days of storage $\left(1.13 \mathrm{meq} \mathrm{kg}^{-1}\right)$ reaching a maximum value of $8.45 \mathrm{meq} \mathrm{kg}^{-1}$ at 90 days (Table 2). Photo-oxidation (4.82 meq $\mathrm{kg}^{-1}$ ) was significantly higher than auto-oxidation $\left(3.80 \mathrm{meq} \mathrm{kg}^{-1}\right)$. The peroxide values were significantly different among the three oils with highest in tobacco seed oil (5.06 meq kg $)$ followed by sunflower oil (4.29 meq kg ${ }^{-1}$ ) and groundnut oil ( $3.58 \mathrm{meq} \mathrm{kg}^{-1}$ ) (Table 2 ), respectively. 
Table 1: Effect of storage period, oxidation methods on chemical properties of tobacco, sunflower and groundnut oils

\begin{tabular}{llllllll}
\hline Source & DF & $\begin{array}{l}\text { Peroxide value } \\
(\mathbf{m e q ~ k g})\end{array}$ & $\begin{array}{l}\text { p-Anisidine } \\
\text { value }\end{array}$ & $\begin{array}{l}\text { Conjugated trienes } \\
\left(\varepsilon^{1 \%} \lambda \mathbf{2 6 8}\right)\end{array}$ & $\begin{array}{l}\text { Conjugated dienes } \\
\left(\varepsilon^{1 \%} \lambda \mathbf{2 3 2}\right)\end{array}$ & $\begin{array}{l}\text { Free fatty } \\
\text { acids }(\mathbf{\%})\end{array}$ & $\begin{array}{l}\text { lodine value } \\
\left(\mathbf{g} \mid \mathbf{1 0 0} \mathbf{g}^{-1}\right)\end{array}$ \\
\hline Replications & 2 & 0.8352 & 0.2317 & 0.0117 & 0.289 & 0.0002 & 2.1666 \\
Treatments & 43 & $25.961^{*}$ & $63.663^{*}$ & $32.396^{*}$ & $72.967^{*}$ & $0.0149^{*}$ & $699.903^{*}$ \\
Days of storage(f1) & 6 & $159.995^{*}$ & $412.73^{*}$ & $217.316^{*}$ & $489.186^{*}$ & $0.0989^{*}$ & $290.013^{*}$ \\
Oxidation methods (f2) & 1 & $33.120^{*}$ & $68.775^{*}$ & $27.188^{*}$ & $45.780^{*}$ & $0.0126^{*}$ & 45.841 \\
Oils (f3) & 2 & $22.817^{*}$ & $48.768^{*}$ & $10.055^{*}$ & $33.666^{*}$ & $0.0063^{*}$ & $14089.7^{*}$ \\
F1.f2 & 6 & $7.216^{*}$ & $7.56^{*}$ & $3.706^{*}$ & $4.3748^{*}$ & $0.0018^{*}$ & 7.8966 \\
F1.f3 & 12 & $1.985^{*}$ & $2.174^{*}$ & $1.172^{*}$ & $4.729^{*}$ & 0.0006 & 4.5251 \\
F2.f3 & 2 & 0.670 & $5.823^{*}$ & 0.5264 & 1.001 & 0.0004 & 2.627 \\
F1.f2.f3 & 12 & 0.621 & 0.939 & $0.373^{*}$ & 0.3143 & 0.0001 & 1.599 \\
Error & 82 & 0.132 & 0.3289 & 0.0888 & 0.2392 & 0.0002 & 10.792 \\
C.V.(\%) & & 8.43 & 8.68 & 10.64 & 9.09 & 9.58 & 2.87 \\
$\mathrm{R}^{2}$ & & 0.990 & 0.990 & 0.9948 & 0.9938 & 0.9761 & 0.971 \\
\hline
\end{tabular}

f1: Storage period; f2: methods of oxidation; f3: oils; *Significant

Table 2 : Interaction effects of storage period, oxidation methods and oil types on chemical properties

\begin{tabular}{|c|c|c|c|c|c|c|}
\hline Days of storage (f1) & $\begin{array}{l}\text { Peroxide value } \\
\text { (meq kg-1) }\end{array}$ & $\begin{array}{l}p \text {-Anisidine } \\
\text { value }\end{array}$ & $\begin{array}{l}\text { Conjugated trienes } \\
\left(\varepsilon^{1 \%} \lambda 268\right)\end{array}$ & $\begin{array}{l}\text { Conjugated dienes } \\
\left(\varepsilon^{1 \%} \lambda 232\right)\end{array}$ & $\begin{array}{l}\text { Free fatty } \\
\text { acids (\%) }\end{array}$ & $\begin{array}{l}\text { lodine value } \\
\left(\mathrm{g} \mid 100 \mathrm{~g}^{-1}\right)\end{array}$ \\
\hline 0 & $0.7022^{\mathrm{a}}$ & $0.6133^{\mathrm{a}}$ & $0.020^{\mathrm{a}}$ & $0.0255^{\mathrm{a}}$ & $0.0794^{\mathrm{a}}$ & $118.777^{\mathrm{a}}$ \\
\hline 15 & $1.1300^{\mathrm{a}}$ & $1.7767^{b}$ & $0.1267^{\mathrm{a}}$ & $1.055^{b}$ & $0.0839^{\mathrm{a}}$ & $117.888^{\mathrm{a}}$ \\
\hline 30 & $2.6333^{b}$ & $4.3233^{\mathrm{c}}$ & $0.3100^{\mathrm{a}}$ & $1.5750^{\mathrm{b}}$ & $0.0933^{\mathrm{ab}}$ & $116.555^{\mathrm{a}}$ \\
\hline 45 & $4.3133^{\circ}$ & $6.7233^{d}$ & $1.1033^{b}$ & $4.0805^{\circ}$ & $0.1122^{\mathrm{ab}}$ & $115.222^{\mathrm{ab}}$ \\
\hline 60 & $5.7955^{d}$ & $8.0333^{\mathrm{e}}$ & $2.9022^{\circ}$ & $6.2711^{\mathrm{d}}$ & $0.1617^{\circ}$ & $112.722^{b}$ \\
\hline 75 & $7.1400^{\circ}$ & $10.9333^{f}$ & $6.3750^{d}$ & $11.2644^{e}$ & $0.2011^{\mathrm{d}}$ & $110.722^{b}$ \\
\hline 90 & $8.4544^{i}$ & $13.8417^{9}$ & $8.7744^{\mathrm{e}}$ & $13.3872^{f}$ & $0.2783^{\mathrm{e}}$ & $107.777^{b}$ \\
\hline \multicolumn{7}{|l|}{ Types of oxidation (f2) } \\
\hline Auto oxidation & $3.7971^{\mathrm{a}}$ & $5.8676^{\mathrm{a}}$ & $2.3371^{\mathrm{a}}$ & $4.7778^{\mathrm{a}}$ & $0.1343^{\mathrm{a}}$ & $114.841^{\mathrm{a}}$ \\
\hline Photo oxidation & $4.8225^{b}$ & $7.3452^{b}$ & $3.2662^{b}$ & $5.9833^{\mathrm{b}}$ & $0.1543^{b}$ & $113.634^{\mathrm{a}}$ \\
\hline \multicolumn{7}{|l|}{ Oils (f3) } \\
\hline Tobacco seed oil & $5.0557^{\mathrm{a}}$ & $7.2748^{\mathrm{a}}$ & $3.2421^{\mathrm{a}}$ & $6.1974^{\mathrm{a}}$ & $0.1526^{a}$ & $129.785^{\mathrm{a}}$ \\
\hline Sunflower oil & $4.2919^{b}$ & $7.1812^{\mathrm{a}}$ & $2.8878^{b}$ & $5.5209^{\mathrm{b}}$ & $0.1500^{\mathrm{a}}$ & $118.880^{b}$ \\
\hline Groundnut oil & $3.5819^{\circ}$ & $5.3633^{b}$ & $2.2750^{c}$ & $4.4233^{\circ}$ & $0.1302^{b}$ & $94.0476^{\mathrm{C}}$ \\
\hline
\end{tabular}

Means having different superscripts within a column are significantly different at $1 \%$

Interaction between days of storage and method of oxidation was significant. The peroxide values for two methods of oxidation for $0,15,30$ and 45 days of storage were at a par. It was observed that photo-oxidation was significantly higher over autooxidation from 60 days on wards and reaching a maximum of 9.80 for photo-oxidation at 90 days (Table 3 ). Interaction between days of storage and oils was highly significant. The peroxide values of three oils at 0 and 15 days of storage were at a par (Table 4). Tobacco seed oil and sunflower oil were at a par for 60,75 and 90 days of storage and were significantly higher than groundnut oil (Table 4). Interaction between oils and oxidation methods was significant. The peroxide values of three oils were significantly different in auto-oxidation and tobacco seed oil had significantly higher values (4.678 meq $\mathrm{kg}^{-1}$ ) followed by sunflower (3.66 meq $\mathrm{kg}^{-1}$ ) and groundnut oil (3.049 meq $\left.\mathrm{kg}^{-1}\right)$. Photo-oxidation of tobacco seed oil and sunflower oil were at a par and significantly higher than groundnut oil (Table 5). Increase in PV on storage has been reported in sunflower oil and canola oil (Neff et al., 1994) and a higher rate of formation of primary oxidation products in light exposed oils (Khan and Shahidi, 2000).

Analysis of variance for $p$-Anisdine value ( $p A V)$ explained $99 \%$ variation with $8.7 \%$ co-efficient of variation. ANOVA for all factors and their interactions were significant at $1 \%$ level (Table 1). The $p A V$ increased significantly with increase in storage period and reaching maximum of 13.84 at 90 days. pAV in photooxidation (7.34) was significantly higher than auto-oxidation (5.87). pAV in tobacco seed oil (7.27) and sunflower oil (7.18) were at par and were significantly higher than groundnut oil (5.36) (Table 2).

Interaction between the storage periods and oxidation methods was significant. Photo-oxidation was significantly higher 
Table 3 : Interaction of storage period (f1) and oxidation methods (f2) on chemical properties of tobacco, sunflower and groundnut oils

\begin{tabular}{|c|c|c|c|c|c|c|}
\hline Factors & $\begin{array}{l}\text { Peroxide value } \\
\left.\text { (meq } \mathrm{kg}^{-1}\right)\end{array}$ & $\begin{array}{l}p \text {-Anisidine } \\
\text { value }\end{array}$ & $\begin{array}{l}\text { Conjugated trienes } \\
\left(\varepsilon^{1 \%} \lambda 268\right)\end{array}$ & $\begin{array}{l}\text { Conjugated dienes } \\
\left(\varepsilon^{1 \%} \lambda 232\right)\end{array}$ & $\begin{array}{l}\text { Free fatty } \\
\text { acids (\%) }\end{array}$ & $\begin{array}{l}\text { lodine value } \\
\left(\mathrm{g} \mid 100 \mathrm{~g}^{-1}\right)\end{array}$ \\
\hline OdxAO & $0.7000^{\mathrm{h}}$ & $0.6133^{j}$ & $0.0200^{\mathrm{h}}$ & $0.0255^{i}$ & $0.0777^{\mathrm{h}}$ & $118.55^{f}$ \\
\hline OdxPO & $0.7044^{h}$ & $0.6133^{j}$ & $0.0200^{h}$ & $0.0255 i$ & $0.08111^{\text {gh }}$ & $119.00^{f}$ \\
\hline $15 \mathrm{dxAO}$ & $1.0800^{\mathrm{h}}$ & $1.5666^{i}$ & $0.1200^{\mathrm{h}}$ & $1.0511^{\mathrm{h}}$ & $0.08111^{\text {gh }}$ & $117.77^{f}$ \\
\hline $15 \mathrm{dxPO}$ & $1.1800^{h}$ & $1.9866^{i}$ & $0.1333^{h}$ & $1.0600^{h}$ & $0.0866^{g h}$ & $118.00^{4}$ \\
\hline $30 \mathrm{dxAO}$ & $2.8533^{9}$ & $3.6000^{h}$ & $0.1533^{h}$ & $1.2300^{\mathrm{gh}}$ & $0.0911^{\text {gh }}$ & $117.00^{\text {ef }}$ \\
\hline $30 \mathrm{dxPO}$ & $2.4133^{9}$ & $5.0466^{9}$ & $0.4667^{\text {gh }}$ & $1.9200^{9}$ & $0.0955^{\text {gh }}$ & $116.11^{\text {def }}$ \\
\hline $45 \mathrm{dxAO}$ & $4.0533^{f}$ & $6.2066^{f}$ & $0.8000^{9}$ & $3.3377^{f}$ & $0.1022^{\text {tg }}$ & $115.55^{\text {def }}$ \\
\hline $45 \mathrm{dxPO}$ & $4.5733^{\text {ef }}$ & $7.2400^{\circ}$ & $1.4067^{\dagger}$ & $4.8322^{\circ}$ & $0.1222^{f}$ & $114.88^{\text {cdef }}$ \\
\hline $60 \mathrm{dxAO}$ & $4.6933^{\mathrm{e}}$ & $7.0000^{\text {ef }}$ & $2.1333^{\mathrm{e}}$ & $5.4400^{\circ}$ & $0.1511^{e}$ & $113.88^{\text {bodef }}$ \\
\hline $60 \mathrm{dxPO}$ & $6.8977^{\circ}$ & $9.0600^{\mathrm{d}}$ & $3.6711^{\mathrm{d}}$ & $7.1022^{d}$ & $0.1722^{\mathrm{de}}$ & $111.55^{\text {abcd }}$ \\
\hline $75 \mathrm{dxAO}$ & $6.0933^{d}$ & $10.226^{c}$ & $5.3133^{c}$ & $10.18^{c}$ & $0.1888^{d}$ & $111.88^{\text {bode }}$ \\
\hline $75 \mathrm{dxPO}$ & $8.1866^{c}$ & $11.640^{b}$ & $7.4367^{\mathrm{b}}$ & $12.34^{b}$ & $0.2133^{\circ}$ & $109.55^{\mathrm{abc}}$ \\
\hline $90 \mathrm{dxAO}$ & $7.1066^{b}$ & $11.853 b^{b}$ & $7.8200^{\mathrm{b}}$ & $12.18^{b}$ & $0.477^{\circ}$ & $109.22^{\mathrm{ab}}$ \\
\hline 90dxPO & $9.8022^{\mathrm{a}}$ & $15.8300^{\mathrm{a}}$ & $9.7200^{\mathrm{a}}$ & $14.59^{\mathrm{a}}$ & $0.3088^{\mathrm{a}}$ & $106.33^{\mathrm{a}}$ \\
\hline
\end{tabular}

d: days;AO:Auto-oxidation; PO: Photo-oxidation; Means having different superscripts within a column are significantly different at $1 \%$

Table 4 : Interaction of storage period ( $\mathrm{f} 1$ ) and oil types ( $\mathrm{f} 3$ ) on chemical properties during storage

\begin{tabular}{|c|c|c|c|c|c|c|}
\hline Factors & $\begin{array}{l}\text { Peroxide value } \\
\text { (meq kg-1) }\end{array}$ & $\begin{array}{l}p \text {-Anisidine } \\
\text { value }\end{array}$ & $\begin{array}{l}\text { Conjugated trienes } \\
\left(\varepsilon^{1 \%} \lambda 268\right)\end{array}$ & $\begin{array}{l}\text { Conjugated dienes } \\
\left(\varepsilon^{11 \%} \lambda 232\right)\end{array}$ & $\begin{array}{l}\text { Free fatty } \\
\text { acids (\%) }\end{array}$ & $\begin{array}{l}\text { lodine value } \\
\left(\mathrm{g} \mid 100 \mathrm{~g}^{-1}\right)\end{array}$ \\
\hline 0dxTO & $0.613^{k}$ & $0.946^{i j}$ & $0.02^{\mathrm{e}}$ & $0.040^{j}$ & $0.081^{\text {tg }}$ & $134.8^{\circ}$ \\
\hline OdxSO & $0.833^{k}$ & $0.653^{i}$ & $0.02^{\circ}$ & $0.026^{j}$ & $0.081^{19}$ & $123.8^{\text {eigh }}$ \\
\hline 0dxGO & $0.660^{k}$ & $0.240^{\circ}$ & $0.02^{\mathrm{e}}$ & $0.010^{j}$ & $0.075^{9}$ & $97.66^{\mathrm{a}}$ \\
\hline $15 \mathrm{dxTO}$ & $1.25^{j^{k}}$ & $2.010^{j k}$ & $0.16^{e}$ & $1.250^{\circ}$ & $0.088^{\text {efg }}$ & $132.6^{\text {cd }}$ \\
\hline $15 \mathrm{dxSO}$ & $1.160^{\mathrm{k}}$ & $2.180^{\text {hk }}$ & $0.12^{\mathrm{e}}$ & $0.980^{\mathrm{ij}}$ & $0.085^{\mathrm{fgi}}$ & $123.0^{\text {fghi }}$ \\
\hline $15 \mathrm{dxGO}$ & $0.980^{k}$ & $1.140^{\mathrm{jk}}$ & $0.10^{\mathrm{e}}$ & $0.936^{i j}$ & $0.078^{9}$ & $98.00^{\mathrm{a}}$ \\
\hline 30dxTO & $3.736^{\prime}$ & $4.946^{f}$ & $0.615^{\mathrm{eg}}$ & $1.591^{i}$ & $0.098^{\text {efg }}$ & $132.5^{\text {cd }}$ \\
\hline 30dxSO & $2.260^{\mathrm{ei}}$ & $4.660^{f}$ & $0.170^{\circ}$ & $1.670^{\mathrm{hi}}$ & $0.093^{\text {efg }}$ & $121.5^{\text {ghij }}$ \\
\hline 30dxGO & $1.903^{i j}$ & $3.360^{\mathrm{h}}$ & $0.145^{\mathrm{e}}$ & $1.460^{\circ}$ & $0.088^{\mathrm{efg}}$ & $95.66^{\mathrm{ab}}$ \\
\hline 45dxTO & $5.950^{d}$ & $7.580^{\mathrm{eg}}$ & $1.170^{\circ}$ & $5.175^{f}$ & $0.115^{\mathrm{de}}$ & $130.3^{\text {cde }}$ \\
\hline $45 \mathrm{dxSO}$ & $4.210^{\mathrm{gl}}$ & $7.270^{\mathrm{eg}}$ & $0.990^{9}$ & $4.413^{f}$ & $0.113^{\text {dei }}$ & $119.6^{\mathrm{hij}}$ \\
\hline $45 \mathrm{dxGO}$ & $2.780^{\circ}$ & $5.32^{\mathrm{df}}$ & $0.610^{\mathrm{eg}}$ & $2.666^{\mathrm{h}}$ & $0.108^{\text {def }}$ & $95.66^{\mathrm{ab}}$ \\
\hline 60dxTO & $6.840^{\mathrm{ch}}$ & $8.31^{\text {bg }}$ & $3.546^{h}$ & $7.210^{9}$ & $0.173^{\mathrm{b}}$ & $129.0^{\text {cdef }}$ \\
\hline $60 \mathrm{dxSO}$ & $5.890^{d}$ & $9.400^{\mathrm{b}}$ & $3.040^{\mathrm{h}}$ & $6.293^{\mathrm{eg}}$ & $0.175^{b}$ & $116.8^{\mathrm{ik}}$ \\
\hline $60 \mathrm{dxGO}$ & $4.6566^{9}$ & $6.390^{\mathrm{de}}$ & $2.120^{\circ}$ & $5.400^{\text {ef }}$ & $0.136^{d}$ & $92.33^{\mathrm{ab}}$ \\
\hline 75dxTO & $7.890^{\mathrm{ab}}$ & $12.060^{\mathrm{a}}$ & $7.230^{\mathrm{af}}$ & $13.330^{d}$ & $0.213^{h}$ & $126.83^{\text {defg }}$ \\
\hline $75 \mathrm{dxSO}$ & $7.286^{\mathrm{bh}}$ & $11.580^{\mathrm{a}}$ & $6.725^{f}$ & $11.113^{\mathrm{a}}$ & $0.211^{h}$ & $115.5^{\mathrm{jk}}$ \\
\hline $75 \mathrm{dxGO}$ & $6.243^{\text {cd }}$ & $8.890^{\mathrm{b}}$ & $5.170^{b}$ & $9.350^{\mathrm{b}}$ & $0.178^{b}$ & $89.83^{b}$ \\
\hline 90dxTO & $9.110^{\prime}$ & $15.070^{\circ}$ & $9.413^{d}$ & $14.875^{\circ}$ & $0.298^{\circ}$ & $122.3^{\text {fohij }}$ \\
\hline 90dxSO & $8.403^{\text {af }}$ & $14.255^{\circ}$ & $9.150^{d}$ & $14.150^{\text {cd }}$ & $0.290^{\circ}$ & $111.8^{k}$ \\
\hline $90 \mathrm{dxGO}$ & $7.850^{\mathrm{ab}}$ & $12.200^{\mathrm{a}}$ & $7.760^{\mathrm{a}}$ & $11.136^{\mathrm{a}}$ & $0.246^{a}$ & $89.16^{b}$ \\
\hline
\end{tabular}

d: days; TO: Tobacco seed oil; SO: Sunflower oil; GO: Groundnut oil; Means having different superscripts within a column are significantly different at $1 \%$

than auto-oxidation from 30 days of storage on wards with maximum increase at 90 days of storage (Table 3). Interaction between storage periods and oils was significant. Generally, it was observed that for each period of storage, tobacco seed oil and sunflower oil were at a par and both were significantly higher than groundnut oil (Table 4). Interaction between oxidation methods and oils was significant. pAV in tobacco seed oil and sunflower oil were at a par for each method of oxidation and was significantly higher than groundnut oil in both oxidation methods (Table 5). $p$-AV is a measure of secondary reaction products that occur during lipid oxidation and it is more robust indicator of the quality of oil and the extent of oxidation. A higher level of $p-A V$ for oil samples exposed to sunlight when compared to stored at ambient may be attributed to the high rate of formation of 
Table 5 : Interaction between oxidation methods (f2) and oil types (f3) on chemical changes during storage

\begin{tabular}{|c|c|c|c|c|c|c|}
\hline Factors & $\begin{array}{l}\text { Peroxide value } \\
\left(\text { meqkg }^{-1}\right)\end{array}$ & $\begin{array}{l}p \text {-Anisidine } \\
\text { value }\end{array}$ & $\begin{array}{l}\text { Conjugated trienes } \\
\left(\varepsilon^{1 \%} \lambda 268\right)\end{array}$ & $\begin{array}{l}\text { Conjugated dienes } \\
\left(\varepsilon^{1 \%} \lambda 232\right)\end{array}$ & $\begin{array}{l}\text { Free fatty } \\
\text { acids (\%) }\end{array}$ & $\begin{array}{l}\text { lodine value } \\
\left.\text { (g|100 } \mathrm{g}^{-1}\right)\end{array}$ \\
\hline AOXTO & $4.678^{b}$ & $6.606^{b}$ & $2.693^{\circ}$ & $5.569^{\circ}$ & $0.143^{b}$ & $130.3^{\mathrm{a}}$ \\
\hline AOxSO & $3.663^{\mathrm{d}}$ & $6.03^{\mathrm{b}}$ & $2.380^{d}$ & $4.778^{d}$ & $0.140^{b}$ & $119.2^{b}$ \\
\hline AOxGO & $3.049^{e}$ & $4.950^{d}$ & $1.937^{\mathrm{e}}$ & $3.986^{e}$ & $0.119^{c}$ & $94.9^{c}$ \\
\hline POXTO & $5.433^{\mathrm{a}}$ & $7.940^{\mathrm{a}}$ & $3.790^{\mathrm{a}}$ & $6.825^{\mathrm{a}}$ & $0.161^{\mathrm{a}}$ & $129.2^{\mathrm{a}}$ \\
\hline POxSO & $4.920^{\mathrm{a}}$ & $8.320^{\mathrm{a}}$ & $3.395^{b}$ & $6.236^{b}$ & $0.159^{\mathrm{a}}$ & $118.5^{b}$ \\
\hline POXGO & $4.114^{\circ}$ & $5.770^{\circ}$ & $2.612^{\text {cd }}$ & $4.860^{d}$ & $0.141^{b}$ & $93.1^{\circ}$ \\
\hline
\end{tabular}

AO: Auto-oxidation; PO: Photo-oxidation; TO: Tobacco seed oil; SO: Sunflower oil; GO: Groundnut oil; Means having different superscripts within a column are significantly different at $1 \%$

secondary oxidation products in the former. Formation of higher levels of secondary oxidation products in oils exposed to light has been reported (Khan and Shahidi, 2002).

Estimation of CD and CT is a good measure of oxidative state of oils (McGinely, 1991). Conjugated trienes content increased with increase in storage period in both oxidation methods in three oils. The analysis of variance for trienes showed significant difference among the means of storage period, oxidation methods and oils at $1 \%$ level of significance. ANOVA for trienes explained $99.5 \%$ variation and the coefficient of variation of $10.6 \%$ which was reasonably small (Table 1 ). The average of three storage periods $0,15,30$ days were at a par, whereas remaining four storage periods viz., 45, 60, 75 and 90 were significantly different among themselves and also with the earlier storage periods (Table 2). Trienes gradually and significantly increased from 45 to 90 days. Triene content was significantly higher in photo-oxidation (3.266) than auto-oxidation (2.337). Three oils were significantly different among themselves. Tobacco seed oil showed significantly higher content of trienes (3.24) over sunflower (2.89) and groundnut oils (2.27) (Table 2). Marcela et al. (2011) reported an increase in conjugated dienes and trienes increased significantly throughout two months storage period of walnut oil.

Interaction of storage periods and methods of oxidation for trienes were significant at $1 \%$ level. The first three storage periods with the methods of oxidations were at a par. From 45 days storage onwards, showed significant variation at each storage period between photo and auto-oxidations. Interaction between storage periods and oils was significant. Treatment combinations at $0,15,30$ days of storage with three oils were not significant. Among most of treatment combinations, tobacco seed oil was at a par with sunflower oil (Table 4). Interaction between oxidation methods and oils was significant. Average content of trienes in auto-oxidation of tobacco seed oil (2.69) and sunflower oil (2.38) were at a par while both of them were significantly higher than groundnut oil (1.94) (Table 5). Similar trend was observed with photo-oxidation. Tobacco seed oil showed 11.62 and $10.42 \%$ higher levels of trienes as compared to sunflower oil in auto and photo-oxidations, respectively. Grujić Slavica et al. (2011) reported an increase in peroxide value, $p$-anisidine of the autoand photooxidized sunflower oil samples and confirmed the decrease in oil quality during storage for 3 months. The PV of virgin olive oil samples increased progressively during the 6 to 8 week storage indicating a greater primary oxidation with a relatively high level of hydroperoxides (Xueqi et al., 2014).

Analysis of variance for conjugated dienes explained $99 \%$ variation and the co-efficient of variation was $9 \%$, which was very small. Conjugated dienes content increased with increase in storage period in both photo and auto-oxidations. Interaction between the storage period, oxidation methods and oils was not significant (Table 1). Zero days of storage showed average conjugated dienes of 0.03 , which was significantly lower than the rest of dates and the highest was 13.38 at 90 days of storage. All the dates of storage were significant except 15 and 30 days. Dienes increased significantly from 45 days onwards with the higher values at 75 and 90 days of storage. Conjugated dienes content in auto-oxidation (4.78) was significantly less than photooxidation (5.98). Tobacco oil had the highest conjugated dienes (6.20) followed by sunflower (5.52) and groundnut oils (4.42) and which were significant among themselves (Table 2).

Interaction between storage periods and oxidation methods was significant. Conjugated diene content was significantly varied from 45 day of storage period onwards between the oxidation methods at each of storage period and photo-oxidation was significantly higher than auto-oxidation (Table 3). Interaction between storage periods and oils was significant. The treatment combination up to 15 days of storage with three oils was at a par. Treatment combination from 30 days of storage with three oils ranging from 1.59 to 14.87 was significant. For each period of storage from 30 days onwards, tobacco seed oil was significantly higher followed by sunflower oil and groundnut oil (Table 4). Among oils, as the storage period increased from 30 days on wards, there was a significant increase in dienes. Treatment combinations for oxidation methods and oils were significant. All three oils were significantly different in autooxidation while in photo-oxidation tobacco seed oil and sunflower 
oil were at par and were significantly higher than groundnut oil. A higher value of conjugated dienes and conjugated trienes in lightexposed oil samples as compared with those stored at ambient might be attributed to the accelerated rate of photo-oxidation and exposure to sunlight radiations that might speed up the oxidative alterations (Pan et al., 2004). Increase in conjugated dienes and conjugated trienes were observed in sunflower oil and soybean oils exposed to different storage conditions (Anwar et al., 2007).

The co-efficient of variation in FFA values was 9.57 and ANOVA has explained $97.6 \%$ of variation. Interaction between storage period and oxidation methods were highly significant, whereas rest of the interactions viz., storage period $x$ oils, and oxidation methods $x$ oils were not significant (Table 1).

Free fatty acids content at 0,15 and 30 days of storage were at a par, whereas 30 and 45 days were also at a par. Free fatty acids significantly increased from 45 days of storage onwards reaching a maximum $(0.278 \%)$ at 90 days (Table 2 ). Free fatty acids content in photo-oxidation $(0.154 \%)$ was significantly higher than auto-oxidation $(0.134 \%)$. Tobacco seed oil $(0.143 \%)$ and sunflower oil $(0.140 \%)$ were at a par and significantly higher than groundnut oil. Both oxidation methods were at a par for different storage periods up to 60 days and were significantly different at 75 and 90 days of storage (Table 2). The PV of virgin olive oil samples increased progressively during the 6 to 8 week storage indicating a greater primary oxidation with a relatively high level of hydroperoxides.

Natural fats and oils are triglyceride form when freshly extracted from the source. On prolonged storage, the triglycerides begin to break down giving rise to free fatty acids due to the presence of moisture in the oil, elevated temperature and, most important of all, lipases (enzyme) coming from the source or contaminating microorganisms (Chandrasekharan, 2013). Presence of excess free fatty acids is an indicator to unnatural state of oil. The present study reveals that the FFA content in tobacco seed oil was at a par with sunflower oil and higher than groundnut oil. Anwar et al. (2007) reported increased FFA content due to oxidation in soybean oil stored at ambient and sun light conditions. Xueqi et al. (2014) reported significant increase in free fatty acids of sunflower oil stored at $25^{\circ} \mathrm{C}$ during the storage period.

The initial iodine values (IV) were 135.2, 123.7 and $98 \mathrm{~g} \mathrm{I}$ $100 \mathrm{~g}^{-1}$ in tobacco seed oil, sunflower oil and groundnut oil respectively. The co-efficient of variation for iodine value was 2.87 which were small indicating small variation among the iodine values. The ANOVA explained $97 \%$ of variability (Table 1). The iodine values decreased from 118.78 to $107.78 \mathrm{gl}_{100 \mathrm{~g}^{-1} \text { with }}$ increase in storage period from 0 to 90 days (Table 2). Only zero days of storage were significantly different with 60,75 and 90 days, while 15 and 30 days of storage were significant with 75 and 90 days. Three oils were significantly different for iodine values.
Tobacco seed oil showed significantly higher value $(129.78 \mathrm{gl}$ $\left.100 \mathrm{~g}^{-1}\right)$ followed by sunflower oil $\left(118.88 \mathrm{gl}^{100 \mathrm{~g}^{-1}}\right)$ and groundnut

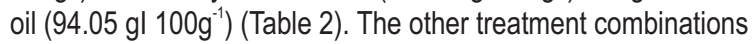
were not significant. lodine value is an indicator of unsaturation and tobacco seed oil showed higher IV indicating the higher level of unsaturated fatty acids. Decrease in IV is an indicator of lipid oxidation (Naz et al., 2004). Tobacco seed oil and sunflower oil showed similar decreasing trends in IVs with increase in storage period.

In the present study, oxidative changes of tobacco seed oil showed nearly similar trends with that of sunflower oil with respect to $p A V$ and FFA, and a marginal increase in conjugated dienes and conjugated trienes in auto-oxidation. Low PV even at 90 days of storage in the present studies indicates that the tobacco seed oil is of good quality. The study suggests that even though tobacco seed oil contains higher levels of unsaturated fatty acids it can be stored like any other edible oils.

\section{References}

Anwar, F., S.A.S. Chatt and A.I. Hussain: Assessment of oxidative deterioration of soybean oil at ambient and sunlight storage. Grasas Y. Aceites, 58, 390-395 (2007).

Awolola, A.G., D.S. Ogunniyi and O. Oluwaniyi: Refining, modification and characterization of tobacco seed oil for its improved potential for industrial use. Nig. J. Pure App. Sci., 23, 2168-2174 (2010).

Chandrasekharan, M.: Valorization of food processing by-products. CRC Press, Taylor \& Francis group. Boca Raton, pp. 35-62 (2013)

Cox, H.E. and D. Pearson: The Chemical Analysis of Foods. Chemical Publishing Co Inc New York, p. 421 (1962).

Deo Singh, K. and C.V. Narasimharao: Tobacco seed oil. In: Alternative uses of tobacco (Eds. K.D. Singh and K.C. Jain). CTRI, Rajahmumdry, pp. 56-63 (2005).

Eunok Choe and B. M. David : Mechanisms and factors for edible oil oxidation. Compr. Rev. Food Sci. Food Saf., 5, 169-186 (2006).

Fekarurhobo, G.K. and F.G. Obomanu: Effects of short-term exposure to sunlight on the quality of some edible vegetable oils. Res J. App. Sci., 4, 152-156 (2009)

Giannelos, P.N., F. Zannikos, S. Stournas, E. Loise and G. Anastopulos: Tobacco seed oil as an alternative diesel fuel: Physical and chemical properties. Indu. Crops Prod., 16,1-9 (2002).

Grujić, S., A. Vojislav and V. Milan: The effect of packing material on storage stability of sunflower oil. Quality of Life, 2, 75-83 (2011).

International Union of Pure and Applied Chemistry (IUPAC): Standard Methods for the Analysis of Oils, Fats and Derivatives, 7th revised and enlarged ed., (Eds.: C. Paquot and A. Hautfenne). Blackwell Scientific, London. (1987).

Khan, M. and F. Shahidi: Rapid oxidation of commercial extra virgin olive oil stored under fluorescent light. J. Food Lipids, 6, 331-339 (2000).

Lowry, R.R. and I.J. Tinsley: Rapid colorimetric determination of free fatty acids. J. Am. Oil Chem. Soc., 53, 470-472 (1976).

Marcela, M, B.A. Guillermina, N. Valeria, G. Nelson and M. Damián aestri: Sensory characterisation and oxidative stability of walnut oil. Int. J. Food Sci. Tech., 46,1276-1281 (2011).

McGinely, L.: Analysis and quality control for processing and processed 
fats. In: Analysis of Oilseeds, Fat and Fatty Food (Eds.: J.B. Rossell and J.L.R. Printed). Elsevier Applied Science, 440-470 (1991).

Mukhtar, A., U. Habib and H. Mukhta: Fatty acid composition of tobacco seed oil and synthesis of alkeyd resins. Chinese J. Chem., 25,705708 (2007).

Naz, S., H. Sheikh, H. Saddiqi and S.A. Syeed: Oxidative stability of olive, corn and soybean oil under different conditions. Food Chem., 88, 235-259 (2004).

Neff, W.E., T.L.Mounts, W.M. Rinsach, H. Konishi and M.A. Elegamy: Oxidative stability of purified canola oil triglycerols with altered fatty acid compositions. J. Amer. Oil Chemists Soc., 71, 215-221(1994).

Pan, X., H. Ushio and T. Ohshima: Photo-oxidation of lipids impregnated on the surface of dried seaweed (Porphyra yezoensis Ueda). Characterization of volatiles. J. Amer. Oil Chemists Soc., 81, 773781(2004).
Pezzuto, J.M. and E.J. Park: Auto-oxidation and anti-oxidation in Swarbrick. In: Encyclopedia of Pharmaceutical Technology (Ed.: J.C. Boylan). Vol. 1, Marcel Deccker Inc. , New York. , 97-113 (2002).

Purseglove, J.W.: Tropical Crops. Longman, England. (1991)

Siva Raju, K., C.V. Narasimha Rao and V. Krishnamurthy: Comparative study on characteristics of seed oil and nutritional composition of seed cake in different Tobacco types cultivated in India. "National Symposium on tobacco-2011", CTRI, Rajahmundry, 20-22, December, 2011, p. 81 (2011).

Xueqi, Li., Hanjiang Zhu, F. Charles, Shoemaker and C.W. Selina: The effect of different cold storage conditions on the compositions of extra virgin olive oil. J. Amer. Oil Chemists Soc., 91,1559-1570 (2014).

Zlatanova, M.M., M. Angelona and G. Antova: Lipid composition of tobacco seeds. Bulg. J. Agric. Sci., 13, 539-544 (2007). 